\title{
Decision Analysis for Risk Management of Firesafety Hazards
}

\section{FRANK NOONAN}

Worcester Polytechnic Institute

Worcester, Massachusetts 01609, USA

\section{ABSTRACT}

Applications of decision analysis in fire protection are reviewed for the purpose of trying to glean collectively from previous studies a design framework for a general purpose decision support system for risk management in fire protection.

\section{INTRODUCTION}

This paper examines the use of decision analysis in the practice of risk management for fire protection within business environments. Risk management, for fire protection, concerns the management of a firm's exposure to losses from fire where solution strategies cover loss prevention, loss transfer and loss retention. As a process risk management has been depicted as involving five steps:

1. identification of risks

2. assessment/measurement of risks

3. generation and evaluation of alternative solutions to risk

4. application of the preferred solution

5. monitoring performance of the solution

Here, only the second and third steps, risk assessment and solution evaluation are of concern.

Decision analysis is a discipline for systematic evaluation of alternative actions as a basis for choosing among them. Decision analysis models often include probabilities that quantify judgements about uncertain future events as well as a utility function which expresses the decision maker's attitudes, or the organization's policies, as regards the assumption of risk (1). The description suggest a strong overlap between the decision analysis discipline and steps 2 and 3 in the risk management process. This paper reviews the literature for applications of decision analysis in fire protection with a view of trying to collectively glean from previous studies a design framework for a general purpose decision support system (DSS) for risk management in fire protection. 
As a final introduction note, it is appropriate to delineate various categories of solution alternatives for fire protection and, also, the general categories of risk or loss that a business operation is exposed due to fire hazards. Loss prevention alternatives can include variations in building design, installing fire walls and automatic detection and suppression systems. Other solutions exist through contingency planning for reducing business interruption risk. There is also loss transfer (i.e. insurance) where a reasonable set of alternatives will examine various lower and upper limits on the amount of loss to be transferred. In measuring risk it is first necessary to estimate the certain or deterministic cost components associated with any solution strategy. These will include one time investment costs and costs which occur periodically over the planning horizon of interest. Second, it is necessary to have procedure for assessing the probabilistic components of loss. The latter, not only includes the direct losses associated with fire damage but, also, business interruption (BI) loss. BI loss can be further decomposed into insurable BI loss and non-insurable (but nonetheless real) market opportunity losses.

\section{LITERATURE REVIEW}

In (2), Shpilberg and DeNeufville present an excellent decision analysis for evaluating investments into fire protection equipment and fire resistive construction vs. the procurement of insurance for both large and small airport facilities. Rather than simply evaluate alternatives in terms of expected loss, exponential utility theory is used and alternatives are compared on the basis of risk adjusted loss. A probability loss function is constructed for each alternative by using historical data on losses from airport fires. However, in using this procedure for risk assessment the authors recognize two significant limitations. First, the historical data base did not include business interruption losses, a fact which may explain the preference (within their study) for insurance over loss prevention investments. Second, they note that the lack of adequate loss statistics preclude examination of a larger, more reasonable, set of loss prevention alternatives.

There are two basic approaches to risk assessment; proabability models can be based on subjective estimates or, as in the case described above, they can be estimated from an empirical data base. When the assessment process is subjective, uncertain events are systematicaliy decomposed into conditional events such that expert judgement can be effectively used in assigning event probabilities (e.g. event or probability trees). In fire protection studies, the practice of risk assessment tends more often to be empirically based. Shpilberg and DeNeufville's study points out that this practice will restrict the number of risk reduction strategies (particularly innovative strategies) that can be evaluated due to the lack of historical statistics reflecting the causal impact of each solution strategy. 
Cozzolino (3) has developed general formulations for risk management in fire protection. He considers loss prevention, loss transfer and loss retention alternatives all in one integrated framework. Like (2), he applies exponential utility theory and uses risk adjusted loss as the performance evaluation measure. However, Cozzolino's work is general and does not really address specific procedure for risk assessment.

Halpern (4) presents an application of decision analysis for choosing between two ways of improving fire protection in a community; he considers the installation of detection alarm systems in dwellings vs. the addition of more fire companies in the community. While Halpern does not apply utility theory and only uses expected loss to evaluate alternatives, his paper is of interest for the procedures used in the assessment of risk. Halpern does use historical data for estimating the probability of loss from a fire. However, when statistics were not available to fully reflect the causal impact of solution strategies, the author forms a hybrid model by merging rational and subjective based models with the empirical results on fire loss.

In (5) Fitzgerald describes the Engineering Method, a general methodology for modeling smoke production and flame movement across spaces and barriers. The Engineering Method uses probability trees to address site specific characteristics with respect to:

- fire growth hazard potential

- barriers

- detection

- automatic suppression

- manual suppression

- distribution of value

and generates a probability function on the percent of physical loss. Fitzgerald's work differs from the previous references in that it describes a general purpose tool focused on the task of risk assessment. Its approach to risk assessment is also different in that it systematically decomposes uncertain events into conditional events which require subjective assessments from the user. In its current stage of development the Engineering Method has a number of limitations. It does not address business interruption loss and it doesn't consider insurance or contingency planning strategies. In its current form, the user interface may involve too many inputs and, also, in its current development there are no built in tools/resources to assist the user in making subjective assessments (e.g. availability of empirical statistics or Bayes Rule methodology). However, the Engineering Method does offer a general purpose tool and is certain to play a role in designing any general DSS for risk management in fire protection. 
In (6) Helzer et al apply decision analysis to evaluate strategies for reducing upholstered furniture fire losses. Like the Engineering Method, the study utilizes probability trees and a subjective framework for risk assessment.

\section{DSS DESIGN GUIDEIINES}

In industry, decision analysis methodology, such as utility theory and subjective probability assessment, is not applied to risk management for fire protection. Risk assessment seems limited to what can be measured from historical results and the selection of a risk management strategy for a given facility is limited to the use of simple, one dimensional, decision rules. For example, "invest in loss prevention only if required by building code or insurance" or "establish contingency plans only if the maximum business interruption loss from a fire incident can exceed \$X". While this type of decision making is attractive for its simplicity and ease of execution one has to question its overall costeffectiveness and wonder whether the practice of risk management for fire protection is not indeed ready for utilizing a DSS which can be comprehensive, situation specific and, yet, reasonable in terms of implementation effort. Assuming this question warrants a positive response, a set of design guidelines for developing a DSS is listed below.

\section{A. Evaluation of Risk}

The evaluation module should formally recognize the existence of risk aversion by evaluating alternatives according to risk adjusted loss rather than expected loss; failure to incorporate risk aversion will lead to under-expenditure for loss prevention and loss transfer. As previously noted, (2) and (3) describe the use of exponential utility for computing risk adjusted loss; (7) provides an excellent review on how to assess a firm's utility function.

B. Insurance Pricing Module

An insurance module is necessary which defines insurance prices and risk reduction constraints, all as a function of any probability loss model on physical and business interruption loss.

C. Risk Assessment

If the DSS is to be both comprehensive and application specific, then the risk assessment process should fully utilize rational models and subjective probability assessment. Loss categories should include physical loss and both insurable and non-insurable business interruption loss. Significant correlation can exist between these categories and the assessment process must consider this coupling. The loss models must be generic with perhaps separate submodules for manufacturing, administrative and warehousing facilities. 
When decision makers are required to assess values for speciflc variables and conditional event probabilities, an expert systems framework should exist where historical statistics and Bayes Rule methodology is available to aid in making assessments. Expert system sub-modules should probably be available on combustibility data and on cost and effectiveness data for both manual and automatic, detection systems and suppression systems. The human interface for assessing risk must be manageable. While subjective techniques are essential to a general purpose DSS, the interface should not require hundreds of inputs from the user. Sensitivity analysis can be employed within the system design, in order to optimize the decomposition of uncertain events and thereby keep assessment requirements to a minimum.

REFERENCES

(1) Brown, Rex V., Andres S. Kahr and Cameron Peterson. Decision Analysis for the Manager. New York: Holt, Rinehart and Winston, Inc., 1974 .

(2) Shpilberg, David and Richard DeNeufville, "Use of Decision Analysis for Optimizing Choice of Fire Protection and Insurance: An Airport Study", Journal of Risk and Insurance, 1975, pp. $133-149$.

(3) Cozzolino, John M., "A Method for the Evaluation of Retained Risk," Journal of Risk and Insurance, 1978, pp. 449-471.

(4) Halpern, Jonathan, "Fire Loss Reduction: Fire Detectors vs. Fire Stations", Management Science, Vol. 25, No. 11, 1979.

(5) Fitzgerald, R.W., "Risk Analysis Using the Engineering Method for Building Firesafety", Center for Firesafety Studies, worcester Polytechnic Institute, 1985.

(6) Helzer, S.G. et al, "Decision Analysis of Strategies for Reducing Upholstered Furniture Fire Losses", NBS Technical Note 1101, U.S. Department of Commerce, National Bureau of Standards, 1979.

(7) Farquhar, P.H., "Utility Assessment Methods", Management Science, vol. 30, No. II, 1984. 
